\title{
Choosing a Better Delay Line Medium between Single-Mode and Multi- Mode Optical Fibers: the Effect of Bending
}

\author{
${ }^{1}$ Federal University Birnin Kebbi \\ Along Kalgo-Bunza Road, PMB 1157, Birnin Kebbi, Kebbi State, Nigeria \\ ${ }^{2}$ Universiti Teknologi Malaysia \\ Sultan Ibrahim Chancellery Building, Johor Bahru, Johor, 81310, Malaysia \\ ${ }^{3}$ Kaduna State University \\ P. M. B 2339, Kaduna State, Nigeria \\ ${ }^{4}$ Universiti Tun Hussein Onn Malaysia \\ 101 Beg Berkunci, Parit Raja, Batu Pahat, Johor, 86400, Malaysia \\ ${ }^{5}$ Federal University of Technology Minna \\ P. M. B. 65, Minna, Nigeria
}

Auwal Mustapha Imam ${ }^{1,2}$, Aliyu Kabiru Isiyaku ${ }^{3,4}$, Mustapha Isah ${ }^{3}$, Mohammed Isah Kimpa ${ }^{4,5}$

DOl: $10.22178 /$ pos.29-1

LCC Subject Category:

TP155-156, QC450-467, QD450-801

Received 17.11.2017

Accepted 10.12.2017

Published online 15.12.2017

Corresponding Author:

Aliyu Kabiru Isiyaku

aikabiru1@gmail.com

(C) 2017 The Authors. This

article is licensed under a

Creative Commons

Attribution 4.0 License

\begin{abstract}
Optical fiber cables are materials whose core is made of silica and other materials such as chalcogenide glasses; they transmit a digital signal via light pulses through an extremely thin strand of glass. The light propagates and is being guided by the core which is surrounded by the cladding. Light travels in the optical fiber in the form of total internal reflection in the core of the fibers. The flexibility, low tensile strength, low signal loss, high bandwidth and other characteristics of optical fibers favors it for use as a delay medium in many applications. Another favorable characteristic of optical fiber delay lines is are their relative insensitivities to environmental effects and electromagnetic interferences. The immunity of optical fibers to interferences and their less weight added advantages to it for use as delay medium. Single-mode and multi-mode are the two most popular types of optical fibers. Single-mode fibers have good propagation and delay properties with a minimal loss that allows the signal to propagate in a large distance with insignificant distortion or attenuation. The percentage of power transmission of single-mode fibers is found to be higher than that of the multi-mode fibers. It is, therefore, a preferred type for use as a delay line. In this paper, relative studies of the two optical fibers modes, and the results of power input/output measurement of the two modes are presented with a view to coming up with a better type for use as a delay medium.
\end{abstract}

Keywords: optical fiber; single-mode fiber; multi-mode fiber; delay line; bandwidth.

\section{INTRODUCTION}

Basically, optical fibers are made up of three parts[15]; the core, cladding and the coating. The core is where the light transmission occurs; it is made of either plastic or glass. A more accurate core is made up of silica. The amount of light transmitted depends on the size of the core. The cladding is a layer of glass that provides a low refractive index at the core interference for reflection within the core. It has different refraction index that allows it to focus and contain the light by reflecting it back into the core, preventing loss. The coating is mostly layers of plastic material applied at the outer of the fiber optics cable for the preservation of fiber strength by providing protection and shock absorption. Its purpose is to increase the strength and add the diameter of the cable. The coating material is mostly colored for identification (Figure 1).

The qualities of a delay medium for application as delay-line can be best understood by considering the parameters such as; bandwidth limitations (dispersion); loss; time delay; linearity and beam propagation factor [42]. 


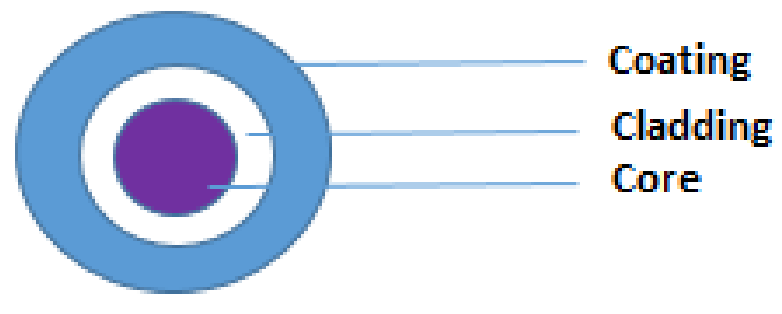

Figure 1 - Basic elements of fiber optics cable

The rise in optical output power from fiber sources through the use of fiber architectures has led to an outstanding performance of optical fibers in terms of output power, beam quality, flexibility, and efficiency. This is achieved due to improved fiber geometry which provides resilience and facilitates efficient conversions of laser beams to high-brightness output [34].

Optical fibers are mostly made from silica, but it is also being made from other materials such as chalcogenide glasses [36]. The optical fibers having advantages such as immunity to electromagnetic interferences and high bandwidth [25] transfer information in the form of light via a process of total internal reflection in the core of the fibers. Optical fiber possesses several favorable features and characteristics that qualify it for use as a delay medium. The flexibility of optical fiber cables allows its construction in small and compact devices. Moreover, provided the optical fiber cable is not bent below a critical bending radius, its flexibility does not give any significant bending losses. The critical bend radius is mostly dependent on the waveguide properties of the optical fiber cable and the wavelength of transmission. Optical fibers have high tensile strength due to their low wall thickness [4].

There has been rapid development of beam quality and efficiency of fiber lasers [10, 34, 52]. Researches K. Wilner and A. P. van den Heuvel [48] report the attractiveness of optical fibers as a delay medium when low loss and dispersion were recorded by using three multi-mode optical fiber delay routes to generate and correlate a 4-bit, 88$\mathrm{Mb} / \mathrm{s}$ coded sequence. Other effective characteristic of optical fiber delay lines are their relative insensitivities to environmental effects and electromagnetic interferences. The immunity of optical fibers to interferences and their less weight is another advantage that favors its application as delay medium.

Dispersion mechanism depends mostly on the waveguide properties of the optical fiber and the optical source spectrum [20]. Different kinds of these dispersions contribute significantly to limiting the bandwidth of the optical fiber [47].

\section{Single-mode Optical Fiber}

Single-mode fiber cables have a single pathway and smaller core diameter that allows only a single wavelength of light to travel [6]. It has small sizes of core and single pathway for light to travel, this enables it to maintain high spatial coherence [51] and constant Gaussian profile of highquality lasers [18]. Due to its small core diameter, the attenuation is decreased because the number of reflections due to light propagations through the core decreases, creating further signal propagation [5].

Single-mode fibers are typically used in connections over a long length. It usually has the diameter of 9/125 for core/cladding. The operating wavelength in single-mode fibers is determined by the cutoff and core diameter. When the wavelength is shorter than the cutoff wavelength, the fiber starts to act like a multi-mode by generating a beam that changes when a fiber is bent and that is not a Gaussian beam. At long wavelength, the core becomes too small to capture the light very well. The transmission then becomes sensitive to the fiber bending and eventually lead to the light not being transmitted by the fiber. One advantage with the single-mode fiber is that the number of the light reflections in the core as the light travels decreases, thereby lowering attenuation and creating the ability for the signal to travel longer.

Due to its low loss $(<0.1 \mathrm{~dB} / \mathrm{ps})$ and large timebandwidth, single-mode optical fiber is an attractive delay medium for processing microwave frequency signals [16]. As a flexible optical path, single-mode optical fibers have often been used in connections in communications, sensing systems, broadband signal processing and configurations in analytical systems. Single-mode fibers have excellent propagation and delay properties with minimal loss and dispersion that allows the signal to propagate in a considerable distance with insignificant distortion or attenuation.

Modal dispersion is absent in single-mode fibers because all the power coupled into single-mode fibers propagates in a single transverse mode as a result, single-mode fibers enables highfrequency signal transmission over a long distance compared to multi-mode fibers [20]. 
Therefore, they (single-mode) are required for devices aimed to provide long delays. The dependence of the fiber refractive index on the wavelength of the optical carrier (material) limits the modulation bandwidth of the single-mode fiber $[27,33]$. The limitations are as a result of the various delays experienced by the other components of the laser light source. But, even though the wavelength of the laser source is not optimal, the material dispersion does not significantly limit the bandwidth of the delay-line devices. Therefore, large or broad bandwidth and long delays can be achieved with single-mode fibers.

\section{Multi-mode Optical Fiber}

Multiple fiber cables have larger core diameter that allows multiple pathways and transmission of several wavelengths of light [3]. Because of this, the number of light reflections as the light propagates through the core increases, making the ability for more data to propagate through the core at a given period [45]. Multi-mode fibers do not maintain high-quality spatial properties of the laser; the light beam is dispersed. The high dispersion rate with multi-mode fiber reduces the quality of the signal over a certain distance [44]. Multi-mode fibers are used mostly for short distance application. Multi-mode fiber exists in two sizes; 50 microns and 62.5 microns. Laser light and LED can be used as a light source for both 50 microns and 62.5 micron cables. Several dispersions limit the bandwidth of optical fiber. In multi-mode fibers, the modulation bandwidth is limited by modal dispersion [20]. Propagation of light through multi-mode optical fiber suffers spatial distortion which leads to scrambled intensity profile [32].

\section{Single-mode and Multi-mode Fibers Power Measurements}

We compared the percentage of transmission of the single-mode and multi-mode fibers by using power meters to determine the input and output powers, thereby exposing the loss due to bending or length of the fiber. The details of the experiment and the results are presented herein.

\section{Experimental setup}

In this paper, we present the optical power input/output measurements of single-mode (SM) 9/125 and multi-mode (MM) 62.5/125 optical fibers using power meters. A YOKOGAWA tunable laser source (TLS) was used at a $1550 \mathrm{~nm}$ wavelength and $7.96 \mathrm{dBm}$ power connected to the optical fibers each of SM and MM. A TLS is a laser that outputs laser light over a predetermined wavelength range [24]. The optical fiber cable was first spliced and cleaved to achieve optimum output. Pigtails were connected to the optical fiber ends and then connected to the TLS at one end and the power meter at the other end. Figure 4 illustrates the process and setup. The optical fibers of $1 \mathrm{~m}$ and $2 \mathrm{~m}$ for both SM and MM were bent with bending diameter of $2 \mathrm{~cm}, 6 \mathrm{~cm}$ and $10 \mathrm{~cm}$ for each to determine the effect of bending on the propagation of signal by measuring the power input and output (Figure 2).

(a)

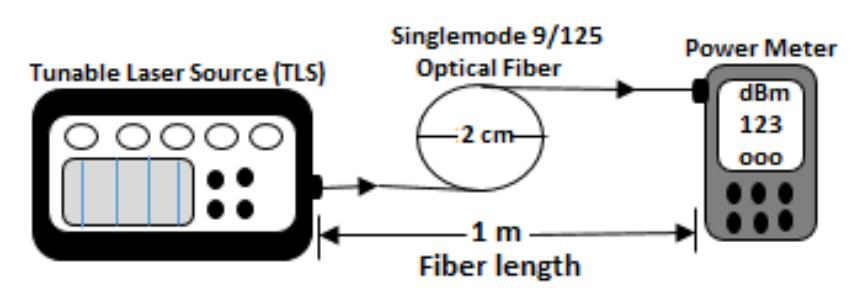

(b)

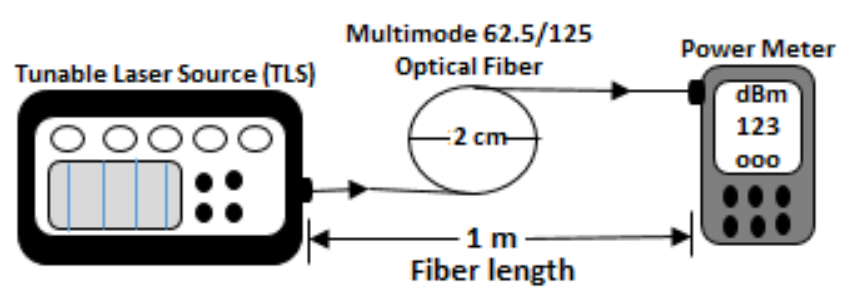

Figure 2 - Schematic diagram of the experimental setup

(a) - Single-mode fiber; (b) - Multi-mode fiber

The modal fields in optical fibers are deformed due to bending such that they become narrower and shift away from an axis along the bending plane; the field shifts create geometrical birefringence [13]. Detailed information about the behavior of optical fibers in many literatures are is based on both numerical simulations and analytical models. Hence, most of the theoretical works are based on optical fibers that are straight [38]. The power input and output of the straight fiber cable for SM and MM were measured at $1 \mathrm{~m}$ and $2 \mathrm{~m}$ lengths. Thereafter, both the lengths were wound and looped at bending diameters of $2 \mathrm{~cm}$, $6 \mathrm{~cm}$ and $10 \mathrm{~cm}$ to determine the effect of the bending at those diameters and the losses due to 
bending of the fibers. The bend magnitude can also be determined by measuring the fiber's core light reduction [14].

\section{Loss (Attenuation)}

Attenuation means loss of light energy as its pulses propagate in the optical cable [22]. It is sometimes called signal loss or fiber loss. An optical signal degrades as it propagates along the optical fiber. Attenuation is directly proportional to the length of the cable and it decides the number of repeaters required from one end to the other [17]. Components such as connectors, splitters, and switches introduce attenuation. Attenuation $\alpha$ is the ratio of the optical output power $P_{o}$ to the input power $P_{i}$ in the fiber of length $L$ :

$$
A=10 \log _{10} \frac{P_{0}}{P_{i}}[\mathrm{~dB} / \mathrm{km}]
$$

Absorption and scattering cause the light energy propagating in an optical fiber to decay exponentially [23]. Scattering is caused by irregularities in core geometry, diameter or improper alterations in the fiber axis direction. Bending and any other dimensional irregularity increases scattering which leads to attenuation. Measuring the fiber attenuation coefficient requires transmitting the light of known wavelength through the fiber and then measure the changes in the distance. Conventionally, fiber can be coupled to a source and then measure the output power at the end, then cut the fiber near the source and measure the power again (Figure 3).
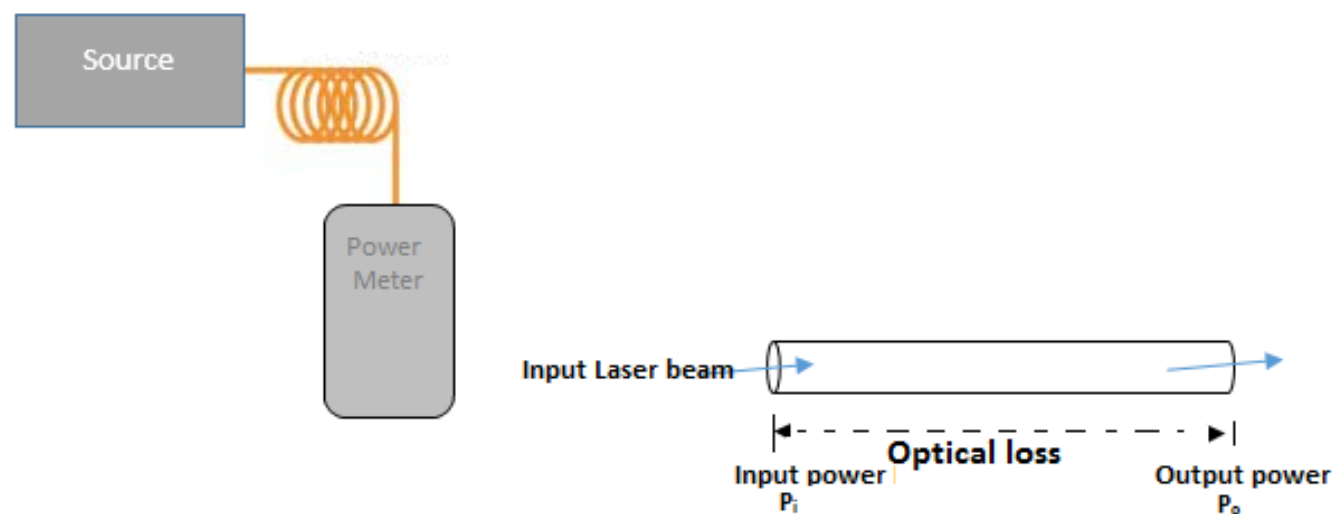

Figure 3 - A set-up of the attenuation measurement in an optical fiber

The most dominant loss mechanism in optical fiber is Rayleigh scattering; it is as a result of the scattering of the propagating electromagnetic wave by little irregularities in the medium [20]. For Rayleigh scattering, the attenuation coefficient per unit length is proportional to $\lambda^{-4}$. Thus, the attenuation is lower at longer wavelength than at shorter wavelength [20].

If the length of the fiber and the power at the end is known, the attenuation coefficient can be determined by calculations via [1]:

Attenuation Coefficient $=\frac{P_{\text {end }}-P_{\text {source }}}{\text { Length }(\mathrm{km})}(\mathrm{dB})$

\section{Bending loss}

The long optical fiber cables involved in delay lines requires wounding into a coil. Bending does not reduce modality in most cases or improve the output beam quality; this is because of the induced mode coupling, modal gain competition and modal deformation [26, 38]. The refractive index of the optical fiber changes when there is a change in density due to bending of the fiber. The refractive index of the optical fiber is equivalent to (3):

$$
n_{\text {material }}^{\prime} \exp \left(\frac{x}{R}\right) \approx n_{\text {material }}^{\prime}\left(1+\frac{x}{R}\right)
$$

where $n_{\text {material }}$ - straight fiber index profile;

$R$ - bend radius;

$x$ - bending orientation [39].

When the fiber is bent, changes occur to the refractive index of the fiber due to optic-stress effect. The refractive index now becomes (4): 


$$
n^{\prime}=n_{\text {material }}\left(1+\frac{\vec{x} \cos \varphi+\vec{y} \sin \varphi}{\rho R}\right)
$$

where $n^{\prime}$ - bent fiber index profile;

$\varphi$ - bending azimuthal angle;

$\rho=$ stress factor.

\section{Leakage loss}

Due to the fact that the refractive index of some part of the cladding is equal to the core, all modes of the fiber suffer from finite leakage losses. This loss is calculated using the relation (5).

$$
\text { Loss }=\frac{40 \pi}{\ln (10) \lambda} I_{m}\left(n_{\text {eff }}\right)
$$

where $n_{\text {eff }}$ is the effective index of the fiber [35].

\section{Coupling light into an optical fiber}

Many applications required that the light emitted from either laser diode or any source be coupled into an optical fiber. The challenges in coupling light into an optical fiber mostly arises from the complicity of the beam characteristics and the fiber core dimension [7], which can be very small. Ball lenses and plano-convex micro-lenses have been developed and applied in coupling light into single-mode optical fiber to enhance the coupling efficiency [8, 21, 37, 43]. Different shapes of lens can be employed to achieve better coupling efficiency $[28,31,41]$. The focused beam characteristics has to match the fiber parameters for excellent coupling efficiency (Figure 4).

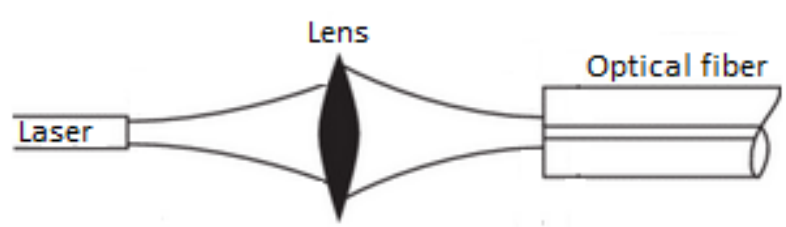

Figure 4 - Schematic illustration of coupling light into an optical fiber

A precise positioning of the fiber to the center of the core in the focused laser beam is required to achieve good coupling efficiency. The coupling efficiency can be tested by measuring the output power of the beam source (laser diode (LD) or tunable laser source (TLS) $P_{\text {beam }}$ without a bicon- vex lens. When the single-mode fiber is mounted on the fiber positioner and aligned, the power of the output beam, $P_{\text {fiber }}$ can then be measured. We followed the same process by measuring the power of the beam before coupling the fiber and the laser source and then measured the power again after coupling. The coupling efficiency $\eta_{\text {coupling }}$ is calculated using [19]:

$$
\eta_{\text {coupling }}=\frac{P_{\text {fiber }}}{P_{\text {beam }}}
$$

To achieve maximum coupling into an optical fiber, the incident field distribution should match that of the fiber mode and the core size should match the focused spot [29]. The core incident angle should not exceed the arcsine of the NA of the optical fiber. A high numerical aperture lens has to collimate the diverging output beam of the laser. M. Hoque [19] introduced a biconvex microlens to maximize coupling of light between an edge emitting laser diode and an optical fiber. The two convex surfaces were with different profiles along their $\mathrm{x}$-and $\mathrm{y}$-axes to enable the elliptical light beam from the laser diode to be fed into the optical fiber core. In this work, we have used pigtails to couple the light from the TLS into the optical fiber.

\section{Optical fiber interconnections}

An interconnection is used when two or more pieces of optical fibers are used to mate them together [40]. The interconnection in optical fiber is practically the use of two connectors or plugs that are aligned in a nose-nose manner and manned with an adapter called coupler. The efficiency of the optical fiber connections depends highly on the connectors and couplers alignment [9]. They are properly aligned and connected tightly in a connector-coupler-connector configuration to minimize loss of light energy propagating within the fiber optics [2].

Accurate and tight connector-coupler-connector alignment reduces the loss of light energy at the interconnection points. Optical fiber of different nature should not be mixed and mated together. For instance, in the multi-mode fiber, if $62.5 \mu \mathrm{m}$ and $50 \mu \mathrm{m}$ are aligned together, some reasonable percentage of the light energy escapes at the interconnection point. Whereas, if the misalignment happens in a single-mode fiber, there may be a total loss of light energy [46]. 


\section{RESULTS AND DISCUSSIONS}

Changes in optical fiber behaviors caused by bending are mostly observed, when perturbation method is applied with regards to the properties of the straight fiber cable [13]. In this work, power meters were used to determine the losses due to bending of both the single-mode and multimode fibers instead of perturbation technique. The percentage of the losses due to bending at some diameters were highlighted. This method works well for single-mode fibers because the field propagation along the single-mode fibers can only be distorted by bending [38]. Table 1 illustrates the results of the measurements of single-mode fiber recorded for 1 meter and 2 meter fibers bent at $2 \mathrm{~cm}, 6 \mathrm{~cm}$ and $10 \mathrm{~cm}$ while Table 2 shows the average total percentage of the transmission.

Some factors that determine propagation between straight and bent fibers show a clear difference between the two.

Table 1 - Single-mode 9/125 Power input/output results with TLS

\begin{tabular}{|l|c|c|c|c|c|c|c|}
\hline S/N & $\begin{array}{c}\text { Optical fiber } \\
\text { Length (m) }\end{array}$ & $\begin{array}{c}\text { TLS Wavelength } \\
(\mathrm{nm})\end{array}$ & $\begin{array}{c}\text { Bending turns } \\
\text { (loop) }\end{array}$ & $\begin{array}{c}\text { Loop } \\
\text { diameter } \\
(\mathrm{cm})\end{array}$ & $\begin{array}{c}\text { Input } \\
\text { Power } \\
(\mathrm{dBm})\end{array}$ & $\begin{array}{c}\text { Output } \\
\text { Power (dBm) }\end{array}$ & $\begin{array}{c}\% \\
\text { Transmission }\end{array}$ \\
\hline 1 & 1 & 1550 & Straight & - & 7.96 & 6.01 & 75.5 \\
\hline 2 & 1 & 1550 & 1 & 2 & 7.96 & 5.72 & 71.9 \\
\hline 3 & 1 & 1550 & 2 & 2 & 7.96 & 5.67 & 71.2 \\
\hline 4 & 1 & 1550 & 3 & 2 & 7.96 & 5.60 & 70.4 \\
\hline 5 & 1 & 1550 & 4 & 2 & 7.96 & 5.51 & 69.2 \\
\hline 6 & 1 & 1550 & 5 & 2 & 7.96 & 5.40 & 67.8 \\
\hline 7 & 1 & 1550 & 1 & 6 & 7.96 & 5.85 & 74.1 \\
\hline 8 & 1 & 1550 & 2 & 6 & 7.96 & 5.80 & 72.9 \\
\hline 9 & 1 & 1550 & 3 & 6 & 7.96 & 5.76 & 72.4 \\
\hline 10 & 1 & 1550 & 4 & 6 & 7.96 & 5.68 & 71.4 \\
\hline 11 & 1 & 1550 & 5 & 6 & 7.96 & 5.60 & 70.4 \\
\hline 12 & 1 & 1550 & 6 & 6 & 7.96 & 5.43 & 68.8 \\
\hline 13 & 1 & 1550 & 1 & 10 & 7.96 & 5.97 & 75.0 \\
\hline 14 & 1 & 1550 & 2 & 10 & 7.96 & 5.85 & 73.5 \\
\hline 15 & 1 & 1550 & 3 & 10 & 7.96 & 5.78 & 72.6 \\
\hline 16 & 1 & 1550 & 4 & 10 & 7.96 & 5.69 & 71.5 \\
\hline 17 & 1 & 1550 & 5 & 10 & 7.96 & 5.64 & 70.9 \\
\hline 18 & 1 & 1550 & 6 & 10 & 7.96 & 5.57 & 70.0 \\
\hline
\end{tabular}

Table 2 - Single-mode 9/125 total percentage of transmission of $1 \mathrm{~m}$ for the bending diameters

\begin{tabular}{|l|c|c|}
\hline $\mathrm{S} / \mathrm{N}$ & Bending Diameter $(\mathrm{cm})$ & \% Transmission \\
\hline 1 & 2 & 59.7 \\
\hline 3 & 6 & 71.7 \\
\hline 3 & 10 & 72.3 \\
\hline
\end{tabular}

Haven calculated the percentage of transmission and the average percentage of transmission as presented in Tables 1 and Table 2, a graph of the total percentage of transmission against the bending diameters is plotted in Figure 5. From the graph, we will see that the effect of diminishing of transmission is less when the bending diameter increases. Even though the bending was not critical, at a very narrow bending of $2 \mathrm{~cm}$ loop diameter, the power output diminished.

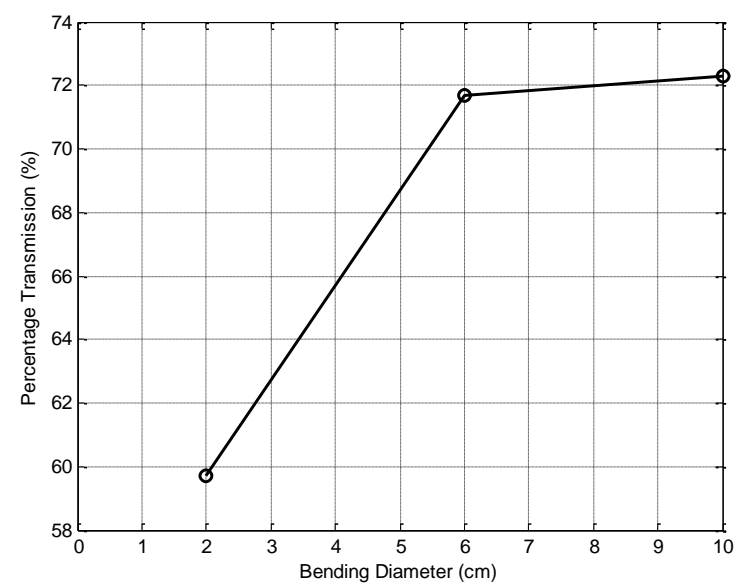

Figure 5 - Graph of the illustration of the percentage transmission against the bending diameters for single mode $9 / 125$ optical fiber 
Table 3 illustrates the results of the measurements of multi-mode fiber recorded for 1 meter and 2 meter fibers bent at $2 \mathrm{~cm}, 6 \mathrm{~cm}$ and $10 \mathrm{~cm}$ while Table 4 shows the average total percentage of the transmission.

Table 3 - Multi-mode 62.5/125 Power input/output results with TLS

\begin{tabular}{|l|c|c|c|c|c|c|c|}
\hline S/N & $\begin{array}{c}\text { Optical fiber } \\
\text { Length (m) }\end{array}$ & $\begin{array}{c}\text { TLS } \\
\text { Wavelength } \\
(\mathrm{nm})\end{array}$ & $\begin{array}{c}\text { Bending } \\
\text { turns (loop) }\end{array}$ & $\begin{array}{c}\text { Loop } \\
\text { diameter } \\
(\mathrm{cm})\end{array}$ & $\begin{array}{c}\text { Input } \\
\text { Power } \\
(\mathrm{dBm})\end{array}$ & $\begin{array}{c}\text { Output } \\
\text { Power } \\
(\mathrm{dBm})\end{array}$ & $\begin{array}{c}\% \\
\text { Transmission }\end{array}$ \\
\hline 1 & 1 & 1550 & Straight & - & 7.96 & 4.32 & 54.1 \\
\hline 2 & 1 & 1550 & 1 & 2 & 7.96 & 3.76 & 47.2 \\
\hline 3 & 1 & 1550 & 2 & 2 & 7.96 & 3.54 & 44.5 \\
\hline 4 & 1 & 1550 & 3 & 2 & 7.96 & 3.31 & 41.6 \\
\hline 5 & 1 & 1550 & 4 & 2 & 7.96 & 3.11 & 39.1 \\
\hline 6 & 1 & 1550 & 5 & 2 & 7.96 & 3.00 & 37.7 \\
\hline 7 & 1 & 1550 & 1 & 6 & 7.96 & 3.79 & 47.6 \\
\hline 8 & 1 & 1550 & 2 & 6 & 7.96 & 3.75 & 47.3 \\
\hline 9 & 1 & 1550 & 3 & 6 & 7.96 & 3.70 & 46.5 \\
\hline 10 & 1 & 1550 & 4 & 6 & 7.96 & 3.53 & 44.3 \\
\hline 11 & 1 & 1550 & 5 & 6 & 7.96 & 3.27 & 41.1 \\
\hline 12 & 1 & 1550 & 6 & 6 & 7.96 & 3.19 & 40.1 \\
\hline 13 & 1 & 1550 & 1 & 10 & 7.96 & 4.27 & 53.6 \\
\hline 14 & 1 & 1550 & 2 & 10 & 7.96 & 4.21 & 52.9 \\
\hline 15 & 1 & 1550 & 3 & 10 & 7.96 & 4.14 & 52.0 \\
\hline 16 & 1 & 1550 & 4 & 10 & 7.96 & 3.89 & 48.9 \\
\hline 17 & 1 & 1550 & 5 & 10 & 7.96 & 3.72 & 46.7 \\
\hline 18 & 1 & 1550 & 6 & 10 & 7.96 & 3.55 & 44.6 \\
\hline
\end{tabular}

es the light wave propagating and radiate away

Table 4 - Multi-mode 62.5/125 total percentage of transmission of $1 \mathrm{~m}$ for the bending diameters

\begin{tabular}{|c|c|c|}
\hline $\mathrm{S} / \mathrm{N}$ & Bending Diameter $(\mathrm{cm})$ & \% Transmission \\
\hline 1 & 2 & 44.0 \\
\hline 3 & 6 & 44.5 \\
\hline 3 & 10 & 49.8 \\
\hline
\end{tabular}

Haven calculated the percentage of transmission and the average percentage of transmission as presented in Tables 3 and Table 4, a graph of the total percentage of transmission against the bending diameters is plotted in Figure 6.

Transition loss and pure bend loss are the two dominant power loss mechanisms in optical fibers, when they are subjected to small radius bend [14]. Transition loss happens at the point between the bent and straight optical fiber segments where the mode fields differ $[11,30]$.

While the pure bend loss occurs in the curved region in which the phase velocity of the fundamental mode is equal to the speed of light in the optical fiber cladding, which subsequently induc-
$[12,49]$.

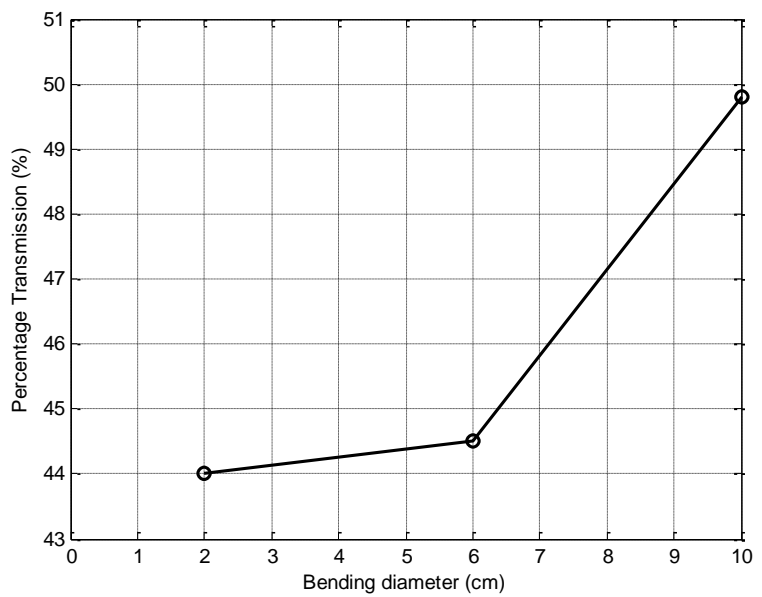

Figure 6 - Graph of the illustration of the average percentage of transmission of the $1 \mathrm{~m}$ multi-mode fiber against the bending diameters

Average of total percentage of transmissions were calculated for the $2 \mathrm{~cm}, 6 \mathrm{~cm}$ and $10 \mathrm{~cm}$ bending diameters to evaluate the effects of bending on the signal transmission in the fiber. 
As the light wave propagates along a fiber by total internal reflection, acoustic waves which induce the periodic fluctuations density in the optical fiber are excited [49]. The backscattered Stokes light is affected by a Doppler shift known as Brillouin frequency shift given by (7):

$$
V_{B}=\frac{2 n_{e f f} V_{A}}{\lambda_{p}}
$$

where $V_{A}=$ acoustic velocity;

$n_{\text {eff }}=$ refractive index of the effective core;

$\lambda_{p}=$ pump wave wavelength [50].

\section{CONCLUSIONS}

Low-loss has provided possibilities of wide-band delay lines using optical fibers. One of the significant characteristics that make an optical fiber unique from other delay-line devices/components is that; the propagation loss of the fiber at a given wavelength is independent of the modulating signal frequency. Bandwidth limitations, small number of taps, difficulties to uniformly illuminate and detect light from a large number of bundled optical fibers, infrared absorption losses at longer wavelength makes multi-mode fibers inefficient for use as delay lines. Even though there are difficulties in obtaining efficient and productive taps in constructing a single mode fiber delay-line, it's extremely large bandwidth, and low signal loss qualifies it as a better delay-line.
Transition loss and pure bend loss are the two dominant power loss mechanisms in optical fibers when they are subjected to small radius bend. Transition loss happens at the point between the bent and straight optical fiber segments where the mode fields differ. While the pure bend loss occurs in the curved region in which the phase velocity of the fundamental mode are equal to the speed of light in the optical fiber cladding. This subsequently induces the light wave propagating and radiate away. Average of the total percentage of transmissions were calculated for the $2 \mathrm{~cm}, 6 \mathrm{~cm}$ and $10 \mathrm{~cm}$ bending diameters to evaluate the effects of bending on the signal transmission in the fiber.

Optimization parameters for a delay-line were elaborated and presented. We have also highlighted some results of the power optimization of the single mode and multi-mode fibers of 1meter and 2-meter-long with bending diameters of $2 \mathrm{~cm}, 6 \mathrm{~cm}$ and $10 \mathrm{~cm}$. A high percentage of transmission was achieved with single-mode fibers compared to multi-mode fibers. Therefore, it is a better media for delay-line as it gives long delay and large bandwidth.

\section{ACKNOWLEDGEMENT}

We acknowledge the support of the Tertiary Education Trust Fund (TETFUND) for supporting our studies in Malaysia and the Laser Centre, Ibnu Sina Institute for Scientific and Industrial Research (ISI-SIR), Universiti Teknologi Malaysia (UTM) for providing us with necessary equipment for research.

\section{REFERENCES}

1. Al-Azzawi, A. (2017). Fiber optics: principles and advanced practices. Boca Raton: CRC Press.

2. Bamiedakis, N., Chen, J., Penty, R. V., \& White, I. H. (2016). High-bandwidth and low-loss multimode polymer waveguides and waveguide components for high-speed board-level optical interconnects. Optical Interconnects XVI. doi: 10.1117/12.2212546

3. Bigot-Astruc, M., Molin, D., Sillard, P., Gooijer, F., \& Jahannes, F. (2013). Bend-resistant multimode optical fiber. Retrieved from http://google.com/patents/EP2642322A1?cl=en

4. Chang, H., Luo, J., Gulgunje, P. V., \& Kumar, S. (2017). Structural and Functional Fibers. Annual Review of Materials Research, 47(1), 331-359. doi: 10.1146/annurev-matsci-120116-114326

5. Colombe, Y., Slichter, D. H., Wilson, A. C., Leibfried, D., \& Wineland, D. J. (2014). Single-mode optical fiber for high-power, low-loss UV transmission. Optics Express, 22(16), 19783. doi:

10.1364/oe.22.019783 
6. Denoyer, G., Cole, C., Santipo, A., Russo, R., Robinson, C., Li, L., ... Vulliet, N. (2015). Hybrid Silicon Photonic Circuits and Transceiver for $50 \mathrm{~Gb} / \mathrm{s}$ NRZ Transmission Over Single-Mode Fiber. Journal of Lightwave Technology, 33(6), 1247-1254. doi: 10.1109/jlt.2015.2397315

7. Dong, L., Li, J., McKay, H., Fu, L., \& Marcinkevicius, A. (2017). Glass large-core optical fibers. Retrieved from https://www.google.ch/patents/US9632243

8. Fadhali, M., Saktioto, Zainal, J., Munajat, Y., Ali, J., \& Abdul Rahman, R. (2008). Mode matching for efficient laser diode to single mode fiber coupling. International Workshop and Conference on Photonics and Nanotechnology 2007. doi: 10.1117/12.799264

9. Fang, W., Lu, M., Liu, X., Gong, L., \& Zhu, Z. (2015). Joint Defragmentation of Optical Spectrum and IT Resources in Elastic Optical Datacenter Interconnections. Journal of Optical Communications and Networking, 7(4), 314. doi: 10.1364/jocn.7.000314

10. Galvanauskas, A. (2004). High Power Fiber Lasers. Optics and Photonics News, 15(7), 42-47. doi: 10.1364/opn.15.7.000042

11. Gambling, W. A., Matsumura, H., \& Ragdale, C. M. (1978). Field deformation in a curved single-mode fibre. Electronics Letters, 14(5), 130. doi: 10.1049/el:19780089

12. Gambling, W. A., Payne, D. N., \& Matsumura, H. (1976). Radiation from curved single-mode fibres. Electronics Letters, 12(21), 567. doi: 10.1049/el:19760431

13. Garth, S. J. (1988). Birefringence in bent single-mode fibers. Journal of Lightwave Technology, 6(3), 445-449. doi: 10.1109/50.4022

14. Gauthier, R. C., \& Ross, C. (1997). Theoretical and experimental considerations for a single-mode fiber-optic bend-type sensor. Applied Optics, 36(25), 6264. doi: 10.1364/ao.36.006264

15. Gong, H., Yang, X., Ni, K., Zhao, C.-L., \& Dong, X. (2014). An Optical Fiber Curvature Sensor Based on Two Peanut-Shape Structures Modal Interferometer. IEEE Photonics Technology Letters, 26(1), 22-24. doi: 10.1109/lpt.2013.2288978

16. Gruner-Nielsen, L., Sun, Y., Jensen, R. V., Nicholson, J. W., \& Lingle, R. (2014). Splicing of few mode fibers. 2014 The European Conference on Optical Communication (ECOC). doi: 10.1109/ecoc.2014.6964087

17. Hecht, J. (2015). Understanding fiber optics. Auburndale: LaserLight Press.

18. Hirose, K., Liang, Y., Kurosaka, Y., Watanabe, A., Sugiyama, T., \& Noda, S. (2014). Watt-class highpower, high-beam-quality photonic-crystal lasers. Nature Photonics, 8(5), 406-411. doi: 10.1038/nphoton.2014.75

19. Hoque, M.-U., Hasan, M. N., \& Lee, Y.-C. (2017). Design and fabrication of a biconvex aspherical microlens for maximizing fiber coupling efficiency with an ultraviolet laser diode. Sensors and Actuators A: Physical, 254, 36-42. doi: 10.1016/j.sna.2016.12.004

20. Jackson, K. P., Newton, S. A., Moslehi, B., Tur, M., Cutler, C. C., Goodman, J. W., \& Shaw, H. J. (1985). Optical Fiber Delay-Line Signal Processing. IEEE Transactions on Microwave Theory and Techniques, 33(3), 193-210. doi: 10.1109/tmtt.1985.1132981

21. Karstensen, H., \& Frankenberger, R. (1989). High-efficiency two lens laser diode to single-mode fiber coupler with a silicon plano convex lens. Journal of Lightwave Technology, 7(2), 244-249. doi: 10.1109/50.17762

22. Kasap, S. O. (2013). Optoelectronics \& photonics: principles \& practices: international edition. N. d: Pearson.

23. Kim, A., Roy, M., Dadani, F., \& Wilson, B. C. (2010). A fiberoptic reflectance probe with multiple source-collector separations to increase the dynamic range of derived tissue optical absorption and scattering coefficients. Optics Express, 18(6), 5580. doi: 10.1364/oe.18.005580

24. Kubota, H. (2005). Tunable laser source. Retrieved from http://bit.ly/2Atm3q0 
25. Lee, B. (2003). Review of the present status of optical fiber sensors. Optical Fiber Technology, 9(2), 57-79. doi: 10.1016/s1068-5200(02)00527-8

26. Li, Z., Zhou, J., Wang, W., He, B., Xue, Y., \& Lou, Q. (2009). Limitations of coiling technique for mode controlling of multimode fiber lasers. 2009 Conference on Lasers \& Electro Optics \& The Pacific Rim Conference on Lasers and Electro-Optics. doi: 10.1109/cleopr.2009.5292572

27. Liu, Z., Tan, Z., Yin, B., Bai, Y., \& Jian, S. (2014). Refractive index sensing characterization of a singlemode-claddingless-singlemode fiber structure based fiber ring cavity laser. Optics Express, 22(5), 5037. doi: 10.1364/oe.22.005037

28. Lu, Y.-K., Tsai, Y.-C., Liu, Y.-D., Yeh, S.-M., Lin, C.-C., \& Cheng, W.-H. (2007). Asymmetric elliptic-coneshaped microlens for efficient coupling to high-power laser diodes. Optics Express, 15(4), 1434. doi: 10.1364/oe.15.001434

29. Luo, Z., Wu, D., Xu, B., Xu, H., Cai, Z., Peng, J., ... Zhang, H. (2016). Two-dimensional material-based saturable absorbers: towards compact visible-wavelength all-fiber pulsed lasers. Nanoscale, 8(2), 1066-1072. doi: 10.1039/c5nr06981e

30. Marcuse, D. (1976). Field deformation and loss caused by curvature of optical fibers. Journal of the Optical Society of America, 66(4), 311. doi: 10.1364/josa.66.000311

31. Modavis, R. A., \& Webb, T. W. (1995). Anamorphic microlens for laser diode to single-mode fiber coupling. IEEE Photonics Technology Letters, 7(7), 798-800. doi: 10.1109/68.393210

32. Morales-Delgado, E. E., Farahi, S., Papadopoulos, I. N., Psaltis, D., \& Moser, C. (2015). Delivery of focused short pulses through a multimode fiber. Optics Express, 23(7), 9109-9120. doi: 10.1364/oe.23.009109

33. Puttnam, B. J., Luis, R. S., Klaus, W., Sakaguchi, J., Delgado Mendinueta, J.-M., Awaji, Y., ... Marciante, J. (2015). $2.15 \mathrm{~Pb} / \mathrm{s}$ transmission using a 22 core homogeneous single-mode multi-core fiber and wideband optical comb. 2015 European Conference on Optical Communication (ECOC). doi: 10.1109/ecoc.2015.7341685

34. Richardson, D. J., Nilsson, J., \& Clarkson, W. A. (2010). High power fiber lasers: current status and future perspectives [Invited]. Journal of the Optical Society of America B, 27(11), B63-B92. doi: $10.1364 /$ josab.27.000b63

35. Saitoh, K., \& Koshiba, M. (2003). Leakage loss and group velocity dispersion in air-core photonic bandgap fibers. Optics Express, 11(23), 3100. doi: 10.1364/oe.11.003100

36. Sanghera, J. ., \& Aggarwal, I. (1999). Active and passive chalcogenide glass optical fibers for IR applications: a review. Journal of Non-Crystalline Solids, 256-257, 6-16. doi: 10.1016/s00223093(99)00484-6

37. Savastru, D., Popescu, M., Miclos, S., Sava, F., Lorinczi, A., Rusu, M., \& Savu, V. (2008). Single mode optical fiber coupling to a laser diode. Optical Memory and Neural Networks, 17(4), 254-257. doi: 10.3103/s1060992x08040024

38. Schermer, R. T. (2007). Mode scalability in bent optical fibers. Optics Express, 15(24), 15674. doi: 10.1364/oe.15.015674

39. Schermer, R. T., \& Cole, J. H. (2007). Improved Bend Loss Formula Verified for Optical Fiber by Simulation and Experiment. IEEE Journal of Quantum Electronics, 43(10), 899-909. doi: 10.1109/jqe.2007.903364

40. Schwaerzle, M., Elmlinger, P., Paul, O., \& Ruther, P. (2015). Miniaturized 3\&\#x00D7;3 optical fiber array for optogenetics with integrated $460 \mathrm{~nm}$ light sources and flexible electrical interconnection. 2015 28th IEEE International Conference on Micro Electro Mechanical Systems (MEMS). doi: 10.1109/memsys.2015.7050911 
41. Shah, V. S., Curtis, L., Vodhanel, R. S., Bour, D. P., \& Young, W. C. (1990). Efficient power coupling from a $980-n m$, broad-area laser to a single-mode fiber using a wedge-shaped fiber endface. Journal of Lightwave Technology, 8(9), 1313-1318. doi: 10.1109/50.59158

42. Sharma, A., \& Kumar, M. (2015). Flat band slow light in silicon photonic crystal waveguide with large delay bandwidth product and low group velocity dispersion. IET Optoelectronics, 9(1), 2428. doi: 10.1049/iet-opt.2014.0090

43. Shiraishi, K., Kagaya, M., Muro, K., Yoda, H., Kogami, Y., \& Tsai, C. S. (2008). Single-mode fiber with a plano-convex silicon microlens for an integrated butt-coupling scheme. Applied Optics, 47(34), 6345. doi: 10.1364/ao.47.006345

44. Sokkar, T. Z. N., Ramadan, W. A., Shams El-Din, M. A., Wahba, H. H., \& Aboleneen, S. S. (2014). Bent induced refractive index profile variation and mode field distribution of step-index multimode optical fiber. Optics and Lasers in Engineering, 53, 133-141. doi: 10.1016/j.optlaseng.2013.09.002

45. Sun, Y., Lingle, R., Shubochkin, R., McCurdy, A. H., Balemarthy, K., Braganza, D., ... Bhoja, S. (2017). SWDM PAM4 Transmission Over Next Generation Wide-Band Multimode Optical Fiber. Journal of Lightwave Technology, 35(4), 690-697. doi: 10.1109/jlt.2016.2618723

46. Tong, X. C. (2014). Advanced materials for integrated optical waveguides. N. d.: Springer.

47. Wang, J., Ashrafi, R., Adams, R., Glesk, I., Gasulla, I., Capmany, J., \& Chen, L. R. (2016). Subwavelength grating enabled on-chip ultra-compact optical true time delay line. Scientific Reports, 6(1). doi: 10.1038/srep30235

48. Wilner, K., \& van den Heuvel, A. P. (1976). Fiber-optic delay lines for microwave signal processing. Proceedings of the IEEE, 64(5), 805-807. doi: 10.1109/proc.1976.10216

49. Xu, P., Dong, Y., Zhang, J., Zhou, D., Jiang, T., Xu, J., ... Bao, X. (2015). Bend-insensitive distributed sensing in singlemode-multimode-singlemode optical fiber structure by using Brillouin optical time-domain analysis. Optics Express, 23(17), 22714. doi: 10.1364/oe.23.022714

50. Zadok, A., Zilka, E., Eyal, A., Thévenaz, L., \& Tur, M. (2008). Vector analysis of stimulated Brillouin scattering amplification in standard single-mode fibers. Optics Express, 16(26), 21692-21707. doi: 10.1364/oe.16.021692

51. Zavalin, A., Yang, J., Haase, A., Holle, A., \& Caprioli, R. (2014). Implementation of a Gaussian Beam Laser and Aspheric Optics for High Spatial Resolution MALDI Imaging MS. Journal of The American Society for Mass Spectrometry, 25(6), 1079-1082. doi: 10.1007/s13361-014-0872-5

52. Zervas, M. N., \& Codemard, C. A. (2014). High Power Fiber Lasers: A Review. IEEE Journal of Selected Topics in Quantum Electronics, 20(5), 219-241. doi: 10.1109/jstqe.2014.2321279 